\title{
PERSONALITY DIFFICULTIES AND RESPONSE TO COMMUNITY-BASED PSYCHOLOGICAL TREATMENT FOR ANXIETY AND DEPRESSION
}

\section{ABSTRACT}

Background: Previous research suggests that comorbid personality disorder may be associated with a less favourable treatment outcome for individuals with depression and anxiety disorder. However, little is known about whether personality difficulties are associated with treatment outcomes within Improving Access to Psychological Therapies (IAPT) services-the largest platform for treating depression and anxiety in England, UK. Secondary aims were to investigate i) whether individual personality difficulties are associated with treatment outcome and ii) whether findings are moderated by treatment type.

Methods: The sample included 3,689 adults who accessed community-based psychological treatment (cognitive behavioural therapy, emotional skills training, or other psychological therapy) for depression and/or anxiety disorder. Associations between personality difficulties (assessed with the Standardised Assessment of Personality-Abbreviated Scale (SAPAS)) and treatment outcomes (recovery and reliable improvement in depression/anxiety symptom scores, assessed using questionnaire-based measures) were investigated using logistic/linear regression.

Results: Personality difficulties were associated with a reduced likelihood of recovery (adjusted OR per unit increase on SAPAS: depression $=0.87,95 \% \mathrm{Cl} 0.84,0.91$; anxiety $=0.86,95 \% \mathrm{Cl} 0.82,0.90)$ and reliable improvement (adjusted OR per unit increase on SAPAS: depression $=0.88,95 \% \mathrm{Cl} 0.84,0.92$; anxiety $=0.85,95 \% \mathrm{Cl} 0.82,0.89)$.. Those with three or more difficulties were over $30 \%$ less likely to recover/reliably improve. 
Limitations: Personality difficulties data were collected via self-report and were not available for all participants.

Conclusion: Patients with personality difficulties have a less favourable response to psychological treatment for depression/anxiety disorder. If replicated, the findings highlight a major challenge to the way community-based psychological therapy services in England (IAPT services) are presently constituted.

Keywords: personality difficulties; psychological therapies; depression; anxiety 


\section{INTRODUCTION}

Depression and anxiety disorders can have a devastating impact on the functioning and quality of life of individuals, and are associated with an increased risk of suicide (Arsenault-Lapierre et al., 2004, Kessler et al., 2014, Bandelow and Michaelis, 2015). Guidelines, such as the National Institute for Health and Care Excellence (NICE), recommend psychological therapies, such as cognitive behavioural therapy (CBT), as an intervention (National Institute for Health and Clinical Excellence, 2011), however, for many patients, timely access to appropriate psychological treatment is limited (Thornicroft et al., 2017, Bandelow and Michaelis, 2015). To help address the increasing burden of common mental health problems in the UK, the Improving Access to Psychological Therapies (IAPT) programme was introduced in England in 2008. IAPT provides evidence-based psychological therapies to people in the community with depression and anxiety disorders. It is recognised as one of the most ambitious programmes of psychological therapies in the world, and currently receives over 1.4 million referrals per annum (Health \& Social Care Information Centre, 2018). In IAPT services, the most commonly delivered treatment is $\mathrm{CBT}$, however other psychological treatments are also available; for example emotional skills training, brief cognitive analytic therapy, or interpersonal therapy.

Two of IAPT's key performance indicators are 'recovery', defined as an individual moving from a 'case' at pre-treatment to a 'non-case' at the end of therapy (based on recommended cutpoints on self-reported measures of depression and anxiety) and 'reliable improvement' (i.e. minimum improvement) in symptom scores (The National Collaborating Centre for Mental Health, 2018). However, only half of patients recover and around two thirds achieve reliable improvement (Health \& Social Care Information Centre, 2018). Identifying the factors that predict an individual's response to treatment would have important implications for clinical practice and could lead to improved tailoring of services and improved outcomes for patients. It has been suggested that the presence of co-morbid personality disorder can lead to less favourable treatment outcomes for those with depression or anxiety disorders (Gorwood et al., 2010, Newton-Howes et al., 2006, Black 
et al., 1994, Hansen et al., 2007, Steketee et al., 2001, Telch et al., 2011, Goddard et al., 2015, Newton-Howes et al., 2014). However, findings are mixed, with some studies reporting no association (Kampman et al., 2008, Joyce et al., 2007, van Bronswijk et al., 2018).

The assessment of personality disorder is lengthy and requires training. In order to allow non-specialists and researchers to rapidly screen for personality disorder, screening tools, such as the Standardized Assessment of Personality - Abbreviated Scale (SAPAS) have therefore been developed. The SAPAS is an 8-item measure refined from the Standardized Assessment of Personality (Mann et al, 1981) which assesses eight core features of PD as described in the ICD-11 and DSM-V, referred to in this paper as 'personality difficulties'. Personality difficulties, as measured by the SAPAS, such as extreme impulsivity or lack of trust, underlie the concept of personality disorder (Morey et al., 2016). Furthermore, other research has shown that the SAPAS captures specific variance associated with personality disorder, as opposed to the variance associated with the "Big Five" personality trait dimensions(Ball et al., 2017). Knowledge of whether there is a relationship between the number of personality difficulties and the effectiveness of treatment for depression and anxiety disorders offered by community-based services such as IAPT is limited. This is an important knowledge gap, as IAPT is the largest platform for treating depression and anxiety disorders in England, and personality difficulties appear to be relatively common among IAPT attenders (Hepgul et al., 2016). Little is also known about the impact of personality difficulties on other key treatment outcomes such as social functioning. This study addressed these knowledge gaps in a in a large sample of patients attending an English IAPT service. The aims of the study were to:

1) Investigate whether levels of personality difficulties were associated with treatment response, including recovery and reliable improvement/deterioration in symptoms of depression and anxiety (adjusting for baseline symptom severity).

2) Investigate whether levels of personality difficulties were associated with change in symptom scores for depression, anxiety, and social functioning. 
3) Identify whether treatment outcomes were differentially associated with different personality difficulties.

4) Explore whether associations with treatment outcomes were moderated by the type of therapy received (CBT, emotional skills training, or other psychological therapy). 


\section{METHODS}

\section{Design and participants}

We conducted an analysis of data obtained from an existing clinical register of IAPT attendees. The sample included adult patients ( $\geq 18$ years) who had accessed the Somerset Partnership NHS Foundation Trust IAPT service for treatment of common mental disorder (depression/anxiety disorders) between 1st November 2012 (when assessment of personality difficulties was first instituted across the service) and $30^{\text {th }}$ April 2018. If a patient presented on more than one occasion during the study period, then only the first instance was included. The Trust has a catchment population of 544,000 and performance levels are slightly higher than the national average with a recovery rate of $56 \%$.

Within IAPT services the most common treatment for depression and anxiety disorders is Cognitive Behavioural Therapy (CBT), however a range of other evidence-based psychological therapies for depression and anxiety disorders are also provided. Treatment is allocated in line with a stepped-care model, which works according to the principle that individuals should be offered the least intrusive treatment appropriate for their needs (The National Collaborating Centre for Mental Health, 2018). Patients with mild-moderate symptoms of depression may receive low-intensity treatments first (such as guided self-help or computerised CBT), whereas those with moderatesevere symptoms of depression may receive high-intensity psychological interventions (such as CBT), delivered by a trained therapist. It is likely that those allocated to high and low intensity treatments differ in important ways including severity of depression/anxiety symptoms, number of personality difficulties, as well as duration and type of therapy received. We therefore restricted our analysis to those who were allocated to high intensity treatment in order to ensure a more homogenous treatment sample $(\mathrm{N}=9,149)$. Treatments received included i) $\mathrm{CBT}$, ii) Emotional Skills Training (EST) (an 8-week emotional skills group program informed by dialectical behaviour therapy), and iii) other psychological therapy (PT) (includes: brief cognitive analytic therapy; humanistic/integrative counselling; brief psychodynamic therapy; eye movement desensitisation and reprocessing; 
interpersonal therapy). Data on personality difficulties was available for $4,733 / 9149$ patients (51.7\%). Those with data recorded on personality difficulties were more likely to be female and to be older than those without. They also differed with regards to their presenting problem, with a higher prevalence of 'mixed depression and anxiety' and 'other' diagnosis (Supplementary Table 1). No differences were found for ethnicity or deprivation score.

The analytical sample was restricted to patients who had the same initial assessment date for depression and anxiety symptoms $(\mathrm{N}=3,819)$. The small number of patients who had data on personality difficulties recorded outside the treatment episode were excluded $(\mathrm{N}=130)$ resulting in a final analytic sample of 3,689 .

\section{Ethical approval}

The authors assert that all procedures contributing to this work comply with the ethical standards of the relevant national and institutional committees on human experimentation and with the Helsinki Declaration of 1975, as revised in 2008. All procedures involving human subjects/patients were approved by the University of Bristol (ref: 62789), and the UK Health Research Authority (IRAS project ref: 229218)

\section{Measures}

The IAPT service follows a standardised protocol and information on symptoms and functional outcomes is routinely collected at each treatment session using validated questionnaire measures (The National Collaborating Centre for Mental Health, 2018). These are not diagnostic tools but demonstrate good diagnostic specificity when compared with clinical interview measures and are routinely used in Health Care settings in the UK. All patients consented to their anonymised information being stored on an electronic database and used for evaluation purposes.

\section{Exposure measure: Number of personality difficulties}


Personality difficulties were assessed using the Standardised Assessment of Personality Abbreviated Scale (SAPAS) (Moran et al., 2003). The SAPAS is an eight-item screen for personality disorder and each item is rated using a dichotomous yes/no format. The total score ranges from 0-8 with higher scores indicating higher likelihood of the presence of personality disorder. The SAPAS has been validated in both clinical (Moran et al, 2003) and population-based samples (Ball et al, 2017; Fok et al, 2015) as well as patients with depression (Bukh et al, 2010; Gorwood et al, 2010). In clinical samples, a score of 3 or more has been found to correctly identify personality disorder in $90 \%$ of patients, with a sensitivity of 0.94 and specificity of 0.85 (Moran et al., 2003). The internal consistency of the SAPAS in the IAPT sample was 0.6, a level consistent with that found in other studies(Fok et al., 2015). As it took time to implement the SAPAS measure into routine practice, it was not recorded at the first assessment for all patients. The proportion who had a SAPAS score recorded at baseline was $30 \%(\mathrm{~N}=1,102)$.

\section{Outcome measures}

\section{1) Engagement with treatment}

A binary variable was created indicating whether participants completed the minimum number of treatment sessions, defined, per service guidelines, as two or more sessions of CBT/PT and four or more sessions of EST.

\section{2) Depressive symptoms (assessed at initial assessment and at last clinical contact)}

Depressive symptoms were assessed using the Patient Health Questionnaire (PHQ-9) (Kroenke and Spitzer, 2002). This is a validated nine-item questionnaire based on the DSM-IV criteria for major depressive disorder and is used to monitor the severity of depression symptoms and response to treatment within IAPT services. It has been shown to be a reliable and valid measure of major depression (Kroenke et al., 2001). Each item is scored from "0" (not at all) to "3" (nearly every day). The maximum score for the PHQ-9 is 27 with higher scores indicating higher levels of impairment. A 
score of 10 or more is used as a cut-off to identify clinical caseness. Patients were classified as 'recovered' if their PHQ-9 scores were above the clinical cut-off at the initial assessment and below the clinical cut-off at the last assessment. A change of 6 or more indicates reliable improvement/deterioration in depression symptoms. The internal consistency of the PHQ-9 in the IAPT sample was 0.8 .

\section{3) Anxiety symptoms (assessed at initial assessment and at last clinical contact)}

Anxiety symptoms were assessed using the Generalised Anxiety Disorder Assessment (GAD-7) (Spitzer et al., 2006). This is a seven-item questionnaire used as a screening tool and severity measure for generalised anxiety disorder (GAD) and is used to monitor the severity of anxiety symptoms and response to treatment within IAPT services. It has been shown to be a reliable and valid measure of GAD (Spitzer et al., 2006). Each item is scored from "0" (not at all) to "3" (nearly every day). The maximum score for the GAD-7 is 21 with higher scores indicating higher levels of impairment. A score of 8 or more is used as a cut-off to identify clinical caseness in IAPT. Patients were classified as 'recovered' if their GAD-7 scores were above the clinical cut-off at the initial assessment and below the clinical cut-off at the last assessment. A change of 4 or more indicates reliable improvement/deterioration in anxiety symptoms in IAPT. The internal consistency of the GAD-7 in the IAPT sample was 0.8 .

Combined depression/anxiety symptoms

As outlined in the IAPT manual (The National Collaborating Centre for Mental Health, 2018), patients were classified as recovered on measures of both depression and anxiety if they scored above the questionnaire cut point on either measure at the first assessment, and below the cutpoint on both questionnaire measures at the last assessment. Patients were classified as having shown reliable improvement if their depression and/or anxiety scores reduced by a reliable amount and neither 
measure showed a reliable increase. Conversely, patients were considered to have reliably deteriorated if their scores for depression and/or anxiety increased by a reliable amount and neither measure showed a reliable decrease.

\section{4) Social functioning (assessed at initial assessment and at last clinical contact)}

Impairment in social functioning was assessed using the Work and Social Adjustment Scale (W\&SAS) (Mundt et al., 2002). This is a five-item scale designed to measure the impact of a patient's perceived mental health difficulties on their functioning (work, home management, social and leisure activities, private leisure activities, and relationships). Each item is scored on an eight-item Likert scale from " 0 " (not at all) to " 8 " (very severely). The maximum score for the W\&SAS is 40 with higher scores indicating higher levels of impairment.

\section{Covariates}

Available covariates included in all models included age, gender, and area level deprivation score. Deprivation deciles are calculated by ranking areas in England from the most deprived to least deprived and dividing into 10 equal groups (higher scores = lower levels of deprivation). Additional covariates used in some analyses included baseline symptom score (for the outcome of interest) and total number of treatment sessions. Information about the specific covariates included in each analysis model are provided in the text/table footnotes.

\section{Statistical Analysis}

We first explored whether personality difficulties were associated with engagement with treatment. The remaining analyses were then restricted to those who completed the minimum number of treatment sessions. Primary outcomes included depression/anxiety recovery and reliable improvement (defined as a minimum reduction in symptoms; 6 for depression and 4 for anxiety). Secondary outcomes included i) change in symptom score between initial assessment and last 
clinical contact (depression, anxiety and social functioning scores) and ii) reliable deterioration in symptoms (an increase of 6 or more symptoms for depression and 4 or more for anxiety).

Associations were explored between the total number of personality difficulties recorded at baseline and each outcome using linear regression (for continuous outcome measures) and logistic regression (for binary outcomes). For the two primary outcome measures (recovery and reliable improvement), we conducted additional analysis to investigate associations with a binary SAPAS score (dichotomised at 3), with individual SAPAS items, and in the sub-sample (30\%) whose SAPAS assessment was completed at the same time as their baseline PHQ-9 and GAD-7 ratings. We also investigated the possibility of effect modification of an association between personality difficulties and treatment response, by treatment received. We did this by testing for an interaction between the number of personality difficulties and treatment type (CBT/EST/PT) in predicting recovery/reliable improvement status, using the likelihood ratio test. All analyses were conducted using STATA version 15. 


\section{RESULTS}

\section{Sample description}

There were 3,689 participants with data on personality difficulties during the treatment episode. SAPAS scores were normally distributed (Supplementary Figure 1) with a mean of 4.3 (SD 1.9). The proportion of the sample endorsing individual SAPAS items ranged from $38.1 \%$ (depends on others a lot) to $84.9 \%$ (normally a worrier). The mean age of the sample was 44.5 years (SD 14.2) and $71.7 \%$ $(n=2,645)$ were female. SAPAS scores were lower in females (difference in SAPAS $-0.37,95 \% \mathrm{Cl}-0.50$ to $-0.24, \mathrm{P}<0.001$ ), in older patients (change in SAPAS per 1-year increase in age $=-0.02,95 \% \mathrm{Cl}-0.03$ to $-0.02, P<0.001$ ) and in those who lived in a less deprived area (change in SAPAS per 1-decile reduction in deprivation $=-0.09,95 \% \mathrm{Cl}-0.12$ to $-0.06, \mathrm{P}<0.001)$. The most common treatment received was CBT ( $n=1,684,45.7 \%)$, with $24.7 \%$ ( $n=912$ ) of the sample receiving EST and $29.6 \%$ $(n=1,092)$ receiving other PT. With regards to treatment allocation, there were no differences in SAPAS scores between those receiving CBT or EST (difference in SAPAS for EST vs CBT $0.10,95 \% \mathrm{Cl}$ 0.05 to $0.25, \mathrm{P}=0.182$ ), however those allocated to 'other PT' had fewer personality difficulties (difference in SAPAS for PT vs CBT $-0.4295 \% \mathrm{Cl}-0.56$ to $-0.28, \mathrm{P}<0.001$; PT vs EST $-0.52,95 \% \mathrm{Cl}-0.68$ to $-0.36, \mathrm{P}<0.001)$.

\section{Association between personality difficulties and baseline symptom scores}

Patients with a greater total number of personality difficulties had higher symptom scores at the first assessment across all measures (Table 1 ).

\section{Missing outcome data}

Outcome data was considered to be missing if the last assessment date for PHQ, GAD and W\&SAS differed by more than 4 weeks. Of the 3,689 patients with SAPAS data, 3625 (98\%) had end of treatment data on at least one outcome variable. The proportion with missing outcome data was $6 \%$ for depression symptoms ( $n=204), 3 \%$ for anxiety symptoms ( $n=126)$, and $20 \%(n=490 / 2,461)$ for 
those with baseline data on social functioning. Compared to those with complete outcome data, patients who were missing data on at least one outcome variable completed fewer treatment sessions, and had a lower baseline score for depression and anxiety (Supplementary Table 2). There were no differences in demographics (age, gender, deprivation), SAPAS score, or baseline social functioning score.

\section{Association between personality difficulties and engagement with treatment}

The number of treatment sessions attended was positively skewed with a mean of 8.4 and a median of 8 sessions (range 1-40). Information on engagement with treatment (completed minimum number of treatment sessions) and relevant covariates (age, gender, deprivation decile, baseline depression and anxiety score) was available for over $99 \%(3,686 / 3,689)$ of patients. Only $7 \%$ failed to engage with treatment ( $n=255 / 3686)$. There was weak evidence to suggest that having a higher SAPAS score was associated with a reduced likelihood of engaging with treatment (OR per unit increase on SAPAS $=1.07,95 \% \mathrm{Cl}: 0.99$ to $1.14, \mathrm{P}=0.070$ ), however the association did not remain following adjustment for covariates $(\mathrm{OR}=1.03,95 \% \mathrm{Cl}=0.95$ to $1.10, \mathrm{P}=0.516)$. The median time between the first and last assessment (for those who completed the minimum number of sessions) was 22 weeks for depression and 24 weeks for anxiety.

\section{Association between personality difficulties and IAPT treatment outcomes}

\section{$\underline{\text { Recovery }}$}

Among the patients who completed the minimum number of treatment sessions, $87 \%(3,002 / 3,433)$ met criteria for clinical caseness (i.e. reached a minimum symptom threshold) for depression at the initial assessment, $89 \%(3,049 / 3,433)$ for anxiety, and $93 \%(3,207 / 3,433)$ for either depression/anxiety. Of these, $48 \%$ of those scoring above the cut-off for depression recovered (i.e. moved from a case to a non-case), $44 \%$ of those scoring above the cut-off for anxiety recovered, and $40 \%$ recovered on both measures. In adjusted analysis (adjusted for age, gender, number of 
sessions, deprivation decile and baseline symptom score) higher SAPAS scores were associated with a reduced likelihood of recovery (Table 2 (OR per unit increase on SAPAS: depression $=0.87,95 \% \mathrm{Cl}$ 0.84 to $0.91, \mathrm{P}<0.001$; anxiety $=0.86,95 \% \mathrm{Cl} 0.82$ to $0.90, \mathrm{P}<0.001$; depression and anxiety $=0.87,95 \%$ $\mathrm{Cl} 0.83$ to $0.91, \mathrm{P}<0.001)$. Table 3 shows associations between personality difficulties and recovery status separately for those receiving CBT, EST and other PT. We did not find statistical evidence for an interaction with treatment type $(\mathrm{P}=0.727$ for depression; $\mathrm{P}=0.585$ for anxiety; $\mathrm{P}=0.472$ for depression and anxiety).

\section{$\underline{\text { Reliable improvement in symptom scores }}$}

Among the patients who completed the minimum number of treatment sessions, $54 \%(1,763 / 3,244)$ had a reliable improvement in depression scores, $59 \%(1,919 / 3,315)$ had a reliable improvement in anxiety scores, and $66 \%(2,107 / 3,184)$ reliably improved on at least one measure. In adjusted analysis (adjusted for age, gender, number of sessions, deprivation decile and baseline symptom score) higher SAPAS scores were associated with a reduced likelihood of reliable improvement (Table 2) (OR per unit increase on SAPAS: depression $=0.88,95 \% \mathrm{Cl} 0.84$ to $0.92, \mathrm{P}<0.001$; anxiety= $0.85,95 \% \mathrm{Cl} 0.82$ to $0.89, \mathrm{P}<0.001$; either depression or anxiety $=0.86,95 \% \mathrm{Cl} 0.82$ to $0.90, \mathrm{P}<0.001$ ). Table 3 shows associations between personality difficulties and reliable improvement status separately for those receiving CBT, EST and other PT. We did not find statistical evidence of an interaction with treatment type $(\mathrm{P}=0.342$ for depression; $\mathrm{P}=0.421$ for anxiety; $\mathrm{P}=0.186$ for depression or anxiety).

\section{Change in symptom scores between first and last assessment}

On average, across the sample, there was improvement in all domains between the first and last assessments (Table 4). In adjusted analyses (adjusted for age, gender, number of sessions and deprivation decile), the change in anxiety and functioning scores was lower for those with a greater number of personality difficulties at baseline (anxiety: $B=-0.10$, standardised beta $=-0.03,95 \% \mathrm{Cl}$ 
-0.06 to $0.01, \mathrm{P}=0.094$; functioning: $B=-0.46$, standardised beta $=-0.09,95 \% \mathrm{Cl}-0.14$ to -0.04 , $P=<0.001)$, however for anxiety, findings did not reach conventional levels of statistical significance. We did not find evidence for an association with depression scores, although the coefficient point estimate was consistent with a reduced change in score with increasing personality difficulties $(B=-$ 0.06 , standardised beta $=-0.02,95 \% \mathrm{Cl}-0.05$ to $0.02, \mathrm{P}=0.360$ ).

\section{Reliable deterioration in symptom scores}

Among the patients who completed the minimum number of treatment sessions, $3 \%$ had a reliable deterioration in depression scores $(98 / 3,244), 6 \%$ had a reliable deterioration in anxiety scores $(187 / 3,315)$, and $6 \%(201 / 3,184)$ had a reliable deterioration on at least one measure. Higher SAPAS scores were associated with an increased likelihood of reliable deterioration (adjusted OR per unit increase on SAPAS: depression=1.20, $95 \% \mathrm{Cl} 1.07$ to $1.36, \mathrm{P}=0.003$; anxiety= $1.23,95 \% \mathrm{Cl} 1.12$ to 1.34, $\mathrm{P}<0.001$; either depression or anxiety $=1.21,95 \% \mathrm{Cl} 1.11$ to $1.32, \mathrm{P}<0.001$ ).

\section{Associations with categorical SAPAS measure}

Additional analyses explored associations between primary outcome measures and a binary SAPAS score, dichotomised at 3, which indicates high risk of personality disorder (Moran et al., 2003). The proportion of the sample that endorsed 3 or more personality difficulties was $81 \%(2,771 / 3,433)$. The proportion with high SAPAS scores was $83 \%$ in the CBT group, $83 \%$ in the EST group and $75 \%$ in the other PT group. Compared to those with low SAPAS scores, those with high scores had lower odds of recovery (adjusted OR per unit increase on SAPAS: depression= $0.64,95 \% \mathrm{Cl} 0.52$ to 0.78 ; anxiety $=0.60,95 \% \mathrm{Cl} 0.49$ to 0.73 ; depression and anxiety $=0.65,95 \% \mathrm{Cl} 0.53$ to 0.79 ) and reliable improvement (adjusted OR per unit increase on SAPAS: depression $=0.73,95 \% \mathrm{Cl} 0.60$ to 0.88 ; anxiety $=0.64,95 \% \mathrm{Cl} 0.53$ to 0.77 ; either depression or anxiety= $0.67,95 \% \mathrm{Cl} 0.55$ to 0.82 ).

\section{Association with individual personality difficulties}


SAPAS items 1 (difficulty making and keeping friends), 2 (usually a loner), 3 (difficulty trusting others), 6 (normally a worrier) and 7 (depends on others a lot) were associated with reduced likelihood of recovery and reliable improvement (Table 5). Patients who endorsed SAPAS items 4 (loses temper easily) and 8 (generally a perfectionist) were also less likely to show reliable improvement.

\section{Sensitivity analysis accounting for differential timing of SAPAS rating}

In order to account for the fact that in the total sample, the timing of SAPAS assessment varied, with only $30 \%$ having synchronised ratings of personality, depression and anxiety symptoms, we conducted a sensitivity analysis in a restricted sample of patients who had SAPAS data recorded at the same time as their baseline measures of depression and anxiety symptoms. The results of this analysis were consistent with the main analysis: compared to those with low SAPAS scores, those with higher scores had lower odds of recovery (adjusted OR per unit increase on SAPAS: depression= $0.88,95 \% \mathrm{Cl} 0.81$ to 0.95 ; anxiety $=0.87,95 \% \mathrm{Cl} 0.81$ to 0.95 ; depression and anxiety $=0.90,95 \% \mathrm{Cl}$ 0.83 to 0.98 ) and reliable improvement (adjusted OR per unit increase on SAPAS: depression $=0.90$, $95 \% \mathrm{Cl} 0.84$ to 0.98 ; anxiety $=0.93,95 \% \mathrm{Cl} 0.86$ to 1.00 ; either depression or anxiety= $0.93,95 \% \mathrm{Cl}$ 0.86 to 1.00). We did not find statistical evidence of an interaction with treatment type in this subsample ( $\mathrm{P}$ value range 0.163 to 0.764$)$. 


\section{DISCUSSION}

We investigated the association between personality difficulties and treatment response in a large sample of IAPT attendees with common mental disorder. Higher SAPAS scores were associated with less favourable treatment outcomes; the odds of recovery and reliable improvement were reduced by $13 \%$ and $14 \%$ respectively for each additional personality difficulty experienced. Those who endorsed high levels of difficulties (3+) were over $30 \%$ less likely to recover or reliably improve. Our findings suggest that while individuals with personality difficulties experience an overall reduction in symptoms following IAPT treatment, the absolute change in anxiety and social functioning scores is reduced. A greater number of personality difficulties was also associated with an increased risk of reliable deterioration in symptoms, although this outcome was experienced by only a small proportion of patients. SAPAS scores were not related to treatment engagement.

\section{Comparison with other studies}

The majority of those allocated to high-intensity IAPT treatments had personality difficulties - $81 \%$ of patients endorsing more than 3 symptoms, indicating high risk of personality disorder. This figure is in line with other data (Hepgul et al., 2016), and suggests that many patients referred to IAPT have complex needs, which may not be adequately addressed with currently available IAPT treatments.

Patients with personality difficulties had more severe psychopathology at baseline with higher depression and anxiety symptoms, and lower levels of social functioning. Yet, even after adjustment for these baseline differences, personality difficulties were associated with poorer outcomes at end of treatment, including a reduced risk of recovery and reliable improvement in symptoms. This is consistent with findings from a meta-analytic review, which found that the presence of co-morbid personality disorder (PD) doubled the likelihood of treatment non-response for depressive disorders (Newton-Howes et al., 2014). Moreover, this association has been found across a variety of different treatment modalities including psychotherapy, antidepressants and 
combination therapy (Newton-Howes et al., 2006, Gorwood et al., 2010). Similar adverse effects of PD on treatment outcome have also been found for patients with anxiety disorders. However, not all findings have been consistent (van Bronswijk et al., 2018, Kampman et al., 2008, Joyce et al., 2007), for example a recent randomised controlled trial found no negative effect of PD on the effectiveness of psychological treatment for patients with Major Depressive Disorder (van Bronswijk et al., 2018).

Our findings are also in line with the only other study of personality difficulties and IAPT treatment response (Goddard et al., 2015). We have shown that this association is also present in a different geographical region, suggesting the existence of a wider phenomenon. Furthermore, we have provided several important extensions to previous work by i) investigating associations with individual personality difficulties, ii) exploring whether there are differential effects according to treatment type, and iii) investigating the effect of personality difficulties on reliable improvement. This latter point is particularly important, as individuals may show improvement in symptoms without moving below the caseness threshold. Furthermore, cases scoring just above the threshold may only decrease by a small amount yet would still be classified as having recovered. Compared with the only other study in this field, our sample is also considerably larger (previous study $n=1249$ ) and treatment was more homogenous, as we exclusively focused on individuals who had been allocated to high intensity treatments delivered by a trained therapist. Further research is needed to investigate whether personality disorders are also associated with treatment response in those patients with milder symptoms, allocated to low intensity treatments.

We did not find evidence of an interaction between personality difficulties and treatment type, suggesting that currently, there is no evidence that a specific type of IAPT treatment is likely to be more beneficial for patients with personality difficulties. This finding needs replication, as our analysis may be underpowered to detect an interaction, however the results are consistent with a recent systematic review (Newton-Howes et al., 2014). Some research has previously suggested that response to particular therapies may be associated with different features of PD; for example Van Bronswijk at el (2018) found that individuals with cluster A PD features responded better to 
cognitive therapy as opposed to interpersonal therapy. Given that the SAPAS is not a diagnostic tool, we were unable to explore differential outcomes according to PD type or cluster of PD in this study. However, we did find that certain personality features were specifically associated with poorer treatment outcome. In particular, those personality difficulties characteristic of cluster A and C disorders (i.e. difficulties with forming and maintaining relationships, being a loner or a worrier, or being dependent on others) were associated with a less favourable treatment outcome. In contrast, we did not find evidence for an association between impulsivity (a trait characteristic of cluster B disorders) and odds of recovery or reliable improvement. Whilst some research suggests that impulsive individuals are more likely to disengage from clinical tasks and thus have worse treatment outcomes(Martino et al., 2012, López-Torrecillas et al., 2014), we found no such association among a large treatment sample presenting for help with common mental disorder. This is important as the finding may help to instil confidence among staff working in IAPT services with patients presenting with impulsive personality characteristics. If replicated, the findings also suggest that screening for the presence of underlying personality difficulties may help IAPT services to establish alternative, more effective care pathways for patients with these difficulties, who inevitably have more complex patterns of relating to practitioners.

\section{Strengths and limitations}

To the best of our knowledge, this is the first study to investigate the relationship between specific personality difficulties and treatment response within the IAPT service, and to explore whether effects are moderated by treatment type. The study involved a large sample of IAPT attendees allocated to high-intensity treatment. Associations were explored with a range of outcomes and analyses were adjusted for relevant confounding variables.

The findings need to be interpreted in light of the following limitations. Firstly, data on personality difficulties were collected via self-report, which could result in an overestimation of pathology when assessed during a depressive episode (Zimmerman, 1994). However, the SAPAS has 
been validated for use in both clinical and population-based samples (Moran et al., 2003, Fok et al., 2015, Hesse and Moran, 2010) and has good diagnostic specificity when compared with clinical interview (Moran et al., 2003). The measure is also practical for use in a busy clinical setting as it is quick to administer. Secondly, differences were found between individuals with and without complete outcome data, and it is possible this may have led to bias. SAPAS data were only available for approximately half of the sample and only $30 \%$ had data recorded at baseline. The low response is due to administrative reasons as it took time to implement the measure into routine practice and it is possible that missing data could introduce bias if those with higher SAPAS scores were more or less likely to complete the questionnaire. Notwithstanding, sensitivity analysis on the subsample with data collected at baseline produced consistent results, supported our main findings. Our analytic sample (those with SAPAS data) also included more female and older patients which may limit the generalisability of our results. Third, although the level of personality difficulties was the same among those allocated to CBT and EST, those allocated to 'other PT' treatment were more likely to have lower SAPAS scores. It is possible that this could influence the results if treatments are not equally effective. Fourth, we adjusted for several key confounders, but residual confounding remains a possibility. Finally, information was not available on other clinically important outcomes such as self-harm and suicidal thoughts. Investigating the relationship between personality difficulties and a broader range of outcomes would be a useful avenue for further research.

\section{Clinical implications}

Personality difficulties are highly prevalent among patients accessing high-intensity treatments within the IAPT service. Our findings suggest that personality difficulties may be associated with treatment outcome and should be routinely assessed before starting treatment. To do so, staff working within IAPT services must be adequately trained in the assessment of individuals with personality difficulties, including an understanding of how these difficulties may impact on clinical presentation and the therapeutic relationship. Our findings suggest that although patients with 
personality difficulties respond to treatment (as indicated by change scores), the extent of improvement in symptoms is reduced. This is particularly the case for patients with 3 or more personality difficulties, who are over $30 \%$ less likely to recover or show reliable improvement in symptoms. Although not a common occurrence ( $6 \%$ of the sample), personality difficulties were also associated with an increased risk of deterioration in symptoms.

Whilst the idea of 'tailoring' IAPT treatment for individuals with personality difficulties may be appealing, the feasibility of achieving this in practice is uncertain. Indeed, given that the commissioning framework for IAPT services is so precisely defined, we would argue that in practice, IAPT services are not set up to provide such tailored treatment. Clearly, identifying the right setting to provide scalable treatment for this large population of individuals remains a huge challenge for the Health service. If replicated, our findings suggest that IAPT services may need to adopt different, change-sensitive outcome measures to better capture treatment response among patients with personality difficulties. Moreover, the intensity of treatment and levels of therapeutic expertise and supervision are likely to require modification if IAPT services are to optimise outcomes for this group of patients.

Acknowledgements: We would like to thank Jane Yeandle (Service Director) and Marc McDonagh (IAPT Service Lead). We also thank Professor David Gunnell (University of Bristol) for his comments on the manuscript.

Funding: This study was funded by the NIHR Biomedical Research Centre at University Hospitals Bristol NHS Foundation Trust and the University of Bristol. The views expressed in this publication are those of the authors and not necessarily those of the NHS, the National Institute for Health Research or the Department of Health and Social Care. The funders had no role in the study design, the collection, analysis and interpretation of data, in the writing of the report or in the decision to submit the article for publication. 
Declaration of Interest: None of the authors report any conflicts of interest

\section{Contributions}

Conception and design of the study: BM, JG, CG, PM; Carrying out the study (including acquisition of study data): BM, JG, CG, PM; Analysis of study data: BM; Interpretation of study data: BM, JG, BDD, CG, JH, DK, NW, PM; Draft the output: BM; Critique the output for important intellectual content: JG, $\mathrm{BDD}, \mathrm{CG}, \mathrm{JH}, \mathrm{DK}, \mathrm{NW}, \mathrm{PM}$. All authors have read and approved the final version of the manuscript.

Data availability: The data that support the findings of this study are available from the Somerset Partnership NHS Foundation Trust IAPT service but restrictions apply to the availability of these data, which were used under license for the current study, and so are not publicly available. 


\section{REFERENCES}

The Improving Access to Psychological Therapies Manual: Appendices and helpful resources [Online]. Available: https://www.england.nhs.uk/wp-content/uploads/2018/06/iapt-manualresources-v2.pdf [Accessed].

ARSENAULT-LAPIERRE, G., KIM, C. \& TURECKI, G. 2004. Psychiatric diagnoses in 3275 suicides: a meta-analysis. BMC psychiatry, 4, 37.

BALL, L., TULLY, R. J. \& EGAN, V. 2017. The SAPAS, personality traits, and personality disorder. Journal of personality disorders, 31, 385-398.

BANDELOW, B. \& MICHAELIS, S. 2015. Epidemiology of anxiety disorders in the 21st century. Dialogues in clinical neuroscience, 17, 327.

BLACK, D. W., WESNER, R. B., GABEL, J., BOWERS, W. \& MONAHAN, P. 1994. Predictors of short-term treatment response in 66 patients with panic disorder. Journal of Affective Disorders, 30, 233-241.

FOK, M. L. Y., SEEGOBIN, S., FRISSA, S., HATCH, S. L., HOTOPF, M., HAYES, R. D. \& MORAN, P. 2015. Validation of the standardised assessment of personality-abbreviated scale in a general population sample. Personality and mental health, 9, 250-257.

GODDARD, E., WINGROVE, J. \& MORAN, P. 2015. The impact of comorbid personality difficulties on response to IAPT treatment for depression and anxiety. Behaviour research and therapy, 73, 1-7.

GORWOOD, P., ROUILLON, F., EVEN, C., FALISSARD, B., CORRUBLE, E. \& MORAN, P. 2010. Treatment response in major depression: effects of personality dysfunction and prior depression. The British Journal of Psychiatry, 196, 139-142.

HANSEN, B., VOGEL, P. A., STILES, T. C. \& GUNNAR GÖTESTAM, K. 2007. Influence of Co-Morbid Generalized Anxiety Disorder, Panic Disorder and Personality Disorders on the Outcome of Cognitive Behavioural Treatment of Obsessive-Compulsive Disorder. Cognitive Behaviour Therapy, 36, 145-155.

HEALTH \& SOCIAL CARE INFORMATION CENTRE. 2018. Psychological Therapies: Annual report on the use of IAPT services. England, 2017-18 [Online]. Available: https://files.digital.nhs.uk/52/D3168F/psych-ther-2017-18-ann-rep.pdf [Accessed 18th February 2019].

HEPGUL, N., KING, S., AMARASINGHE, M., BREEN, G., GRANT, N., GREY, N., HOTOPF, M., MORAN, P., PARIANTE, C. M. \& TYLEE, A. 2016. Clinical characteristics of patients assessed within an Improving Access to Psychological Therapies (IAPT) service: results from a naturalistic cohort study (Predicting Outcome Following Psychological Therapy; PROMPT). BMC psychiatry, 16, 52.

HESSE, M. \& MORAN, P. 2010. Screening for personality disorder with the Standardised Assessment of Personality: Abbreviated Scale (SAPAS): further evidence of concurrent validity. BMC psychiatry, 10, 10.

JOYCE, P. R., MCKENZIE, J. M., CARTER, J. D., RAE, A. M., LUTY, S. E., FRAMPTON, C. M. \& MULDER, R. T. 2007. Temperament, character and personality disorders as predictors of response to interpersonal psychotherapy and cognitive-behavioural therapy for depression. The British Journal of Psychiatry, 190, 503-508.

KAMPMAN, M., KEIJSERS, G. P., HOOGDUIN, C. A. \& HENDRIKS, G.-J. 2008. Outcome prediction of cognitive behaviour therapy for panic disorder: Initial symptom severity is predictive for treatment outcome, comorbid anxiety or depressive disorder, cluster $\mathrm{C}$ personality disorders and initial motivation are not. Behavioural and Cognitive Psychotherapy, 36, 99-112.

KESSLER, R. C., DE JONGE, P., SHAHLY, V., VAN LOO, H. M., WANG, P. S.-E. \& WILCOX, M. A. 2014. Epidemiology of depression.

KROENKE, K. \& SPITZER, R. L. 2002. The PHQ-9: a new depression diagnostic and severity measure. Psychiatric annals, 32, 509-515. 
KROENKE, K., SPITZER, R. L. \& WILLIAMS, J. B. 2001. The PHQ-9: validity of a brief depression severity measure. Journal of general internal medicine, 16, 606-613.

LÓPEZ-TORRECILLAS, F., PERALES, J. C., NIETO-RUIZ, A. \& VERDEJO-GARCÍA, A. 2014. Temperament and impulsivity predictors of smoking cessation outcomes. PloS one, 9, e112440.

MARTINO, F., MENCHETTI, M., POZZI, E. \& BERARDI, D. 2012. Predictors of dropout among personality disorders in a specialist outpatients psychosocial treatment: a preliminary study. Psychiatry and clinical neurosciences, 66, 180-186.

MORAN, P., LEESE, M., LEE, T., WALTERS, P., THORNICROFT, G. \& MANN, A. 2003. Standardised Assessment of Personality-Abbreviated Scale (SAPAS): preliminary validation of a brief screen for personality disorder. The British Journal of Psychiatry, 183, 228-232.

MOREY, L., BENSON, K. \& SKODOL, A. 2016. Relating DSM-5 section III personality traits to section II personality disorder diagnoses. Psychological Medicine, 46, 647.

MUNDT, J. C., MARKS, I. M., SHEAR, M. K. \& GREIST, J. M. 2002. The Work and Social Adjustment Scale: a simple measure of impairment in functioning. The British Journal of Psychiatry, 180, 461-464.

NATIONAL INSTITUTE FOR HEALTH AND CLINICAL EXCELLENCE 2011. Common mental health disorders-identification and pathways to care: NICE clinical guideline. Available from https://www.nice.org.uk/guidance/cg123/evidence/full-guideline-pdf-181771741.

NEWTON-HOWES, G., TYRER, P. \& JOHNSON, T. 2006. Personality disorder and the outcome of depression: meta-analysis of published studies. The British Journal of Psychiatry, 188, 13-20.

NEWTON-HOWES, G., TYRER, P., JOHNSON, T., MULDER, R., KOOL, S., DEKKER, J. \& SCHOEVERS, R. 2014. Influence of personality on the outcome of treatment in depression: systematic review and meta-analysis. Journal of Personality Disorders, 28, 577-593.

SPITZER, R. L., KROENKE, K., WILLIAMS, J. B. \& LÖWE, B. 2006. A brief measure for assessing generalized anxiety disorder: the GAD-7. Archives of internal medicine, 166, 1092-1097.

STEKETEE, G., CHAMBLESS, D. L. \& TRAN, G. Q. 2001. Effects of axis I and II comorbidity on behavior therapy outcome for obsessive-compulsive disorder and agoraphobia. Comprehensive Psychiatry, 42, 76-86.

TELCH, M. J., KAMPHUIS, J. H. \& SCHMIDT, N. B. 2011. The effects of comorbid personality disorders on cognitive behavioral treatment for panic disorder. Journal of psychiatric research, 45 , 469-474.

THE NATIONAL COLLABORATING CENTRE FOR MENTAL HEALTH. 2018. The Improving Access to Psychological Therapies Manual, [Online]. Available: https://www.england.nhs.uk/wpcontent/uploads/2018/06/the-iapt-manual.pdf [Accessed 17/12/2018].

THORNICROFT, G., CHATTERJI, S., EVANS-LACKO, S., GRUBER, M., SAMPSON, N., AGUILAR-GAXIOLA, S., AL-HAMZAWI, A., ALONSO, J., ANDRADE, L. \& BORGES, G. 2017. Undertreatment of people with major depressive disorder in 21 countries. The British Journal of Psychiatry, 210, 119-124.

VAN BRONSWIJK, S. C., LEMMENS, L. H., VIECHTBAUER, W., HUIBERS, M. J., ARNTZ, A. \& PEETERS, F. P. 2018. The impact of personality disorder pathology on the effectiveness of cognitive therapy and interpersonal psychotherapy for major depressive disorder. Journal of affective disorders, 225, 530-538.

ZIMMERMAN, M. J. A. O. G. P. 1994. Diagnosing personality disorders: A review of issues and research methods. 51, 225-245. 
Table 1: Association between SAPAS score and baseline symptom scores

\begin{tabular}{|c|c|c|c|c|c|c|}
\hline & \multicolumn{3}{|c|}{ Unadjusted } & \multicolumn{3}{|c|}{ Adjusted } \\
\hline & $B(95 \% C l)$ & $\begin{array}{l}\text { Standardised } \\
\text { Beta }(95 \% \mathrm{Cl})\end{array}$ & $P$ & $B(95 \% C I)$ & $\begin{array}{l}\text { Standardised } \\
\text { Beta }(95 \% \mathrm{Cl})\end{array}$ & $P$ \\
\hline Depression score $(n=3687)$ & $0.92(0.83,1.02)$ & $0.30(0.26,0.33)$ & $<0.001$ & $0.96(0.86,1.06)$ & $0.31(0.27,0.34)$ & $<0.001$ \\
\hline Anxiety score ( $n=3687)$ & $0.79(0.71,0.87)$ & $0.30(0.27,0.33)$ & $<0.001$ & $0.79(0.71,0.87)$ & $0.30(0.27,0.34)$ & $<0.001$ \\
\hline Functioning score $(n=2,460)$ & $1.12(0.95,1.29)$ & $0.25(0.21,0.28)$ & $<0.001$ & $1.13(0.96,1.31)$ & $0.25(0.21,0.29)$ & $<0.001$ \\
\hline
\end{tabular}

Adjusted for age, gender and deprivation decile

Unstandardised coefficients represents the change in dependent variable per 1-unit increase in SAPAS score; standardised coefficients represent the SD change in dependent variable per SD change in SAPAS score 
Table 2: Association between SAPAS score, recovery and reliable improvement

\begin{tabular}{|c|c|c|c|c|}
\hline Treatment Outcomes & $\begin{array}{l}\text { Unadjusted } \\
\text { OR }(95 \% \mathrm{Cl})\end{array}$ & P Value & $\begin{array}{l}\text { Adjusted } \\
\text { OR }(95 \% \mathrm{Cl})\end{array}$ & P Value \\
\hline $\begin{array}{l}\text { Recovery } \dagger \\
\text { Depression }(n=3,000) \\
\text { Anxiety }(n=3,047) \\
\text { Depression and anxiety }(n=3,205) \ddagger\end{array}$ & $\begin{array}{l}0.82(0.79,0.85) \\
0.81(0.78,0.85) \\
0.80(0.77,0.84)\end{array}$ & $\begin{array}{l}<0.001 \\
<0.001 \\
<0.001\end{array}$ & $\begin{array}{l}0.87(0.84,0.91) \\
0.86(0.82,0.90) \\
0.87(0.83,0.91)\end{array}$ & $\begin{array}{l}<0.001 \\
<0.001 \\
<0.001\end{array}$ \\
\hline $\begin{array}{l}\text { Reliable improvement } \\
\text { Depression }(n=3,242) \\
\text { Anxiety }(n=3,313) \\
\text { Depression or anxiety }(n=3,182)\end{array}$ & $\begin{array}{l}0.97(0.94,1.01) \\
0.94(0.91,0.98) \\
0.93(0.90,0.97)\end{array}$ & $\begin{array}{l}0.147 \\
0.001 \\
0.001\end{array}$ & $\begin{array}{l}0.88(0.84,0.92) \\
0.85(0.82,0.89) \\
0.86(0.82,0.90)\end{array}$ & $\begin{array}{l}<0.001 \\
<0.001 \\
<0.001\end{array}$ \\
\hline
\end{tabular}

Adjusted for age, gender, number of sessions, deprivation decile and baseline score (of outcome variable). $n=2$ with missing data on confounders † Association with recovery among those meeting criteria for clinical caseness at first assessment

$\ddagger$ Recovery among those meeting criteria for clinical caseness for either depression or anxiety at first assessment

OR represents the odds of outcome per 1-unit increase in SAPAS score 
Table 3: Association between SAPAS score, recovery and reliable improvement according to treatment group

\begin{tabular}{|c|c|c|c|c|c|c|}
\hline \multirow[b]{2}{*}{ Treatment Outcomes } & \multicolumn{2}{|c|}{$\begin{array}{l}\text { Cognitive Behavioural } \\
\text { Therapy }\end{array}$} & \multicolumn{2}{|c|}{ Emotional Skills Training } & \multicolumn{2}{|c|}{$\begin{array}{l}\text { Other Psychological } \\
\text { Therapy }\end{array}$} \\
\hline & $\begin{array}{l}\text { Adjusted } \\
\text { OR }(95 \% \mathrm{Cl})\end{array}$ & P Value & $\begin{array}{l}\text { Adjusted } \\
\text { OR }(95 \% \mathrm{Cl})\end{array}$ & P Value & $\begin{array}{l}\text { Adjusted } \\
\text { OR }(95 \% \mathrm{Cl})\end{array}$ & P Value \\
\hline Recovery $\dagger$ & & & & & & \\
\hline Depression & $0.88(0.83,0.94)$ & $<0.001$ & $0.88(0.80,0.96)$ & 0.006 & $0.86(0.79,0.93)$ & $<0.001$ \\
\hline Anxiety & $0.85(0.79,0.90)$ & $<0.001$ & $0.89(0.81,0.97)$ & 0.011 & $0.85(0.79,0.92)$ & $<0.001$ \\
\hline Depression and anxiety $\ddagger$ & $0.86(0.81,0.92)$ & $<0.001$ & $0.90(0.82,0.99)$ & 0.030 & $0.86(0.79,0.93)$ & $<0.001$ \\
\hline Reliable improvement & & & & & & \\
\hline Depression & $0.84(0.78,0.89)$ & $<0.001$ & $0.93(0.85,1.02)$ & 0.110 & $0.91(0.85,0.98)$ & 0.013 \\
\hline Anxiety & $0.83(0.78,0.89)$ & $<0.001$ & $0.91(0.84,0.99)$ & 0.040 & $0.84(0.78,0.91)$ & $<0.001$ \\
\hline Depression or anxiety & $0.82(0.77,0.88)$ & $<0.001$ & $0.92(0.84,1.02)$ & 0.101 & $0.87(0.81,0.94)$ & $<0.001$ \\
\hline
\end{tabular}

Adjusted for age, gender, number of sessions, deprivation decile and baseline score (of outcome variable). $n=2$ with missing data on confounders $\dagger$ Association with recovery among those meeting criteria for clinical caseness at first assessment

‡ Recovery among those meeting criteria for clinical caseness for either depression or anxiety at first assessment

OR represents the odds of outcome per 1-unit increase in SAPAS score 
Table 4: Association between SAPAS score and change in symptom score between first and last assessment

\begin{tabular}{|c|c|c|c|c|c|c|c|c|}
\hline \multirow[t]{2}{*}{ Treatment Outcomes † } & \multirow{2}{*}{$\begin{array}{l}\text { Score at } \\
\text { first } \\
\text { assessment }\end{array}$} & \multirow{2}{*}{$\begin{array}{l}\text { Score at last } \\
\text { assessment }\end{array}$} & \multicolumn{3}{|c|}{ Unadjusted } & \multicolumn{3}{|c|}{ Adjusted } \\
\hline & & & $B(95 \% C l)$ & $\begin{array}{l}\text { Standardised } \\
\text { Beta }(95 \% \mathrm{CI})\end{array}$ & $\begin{array}{l}P \\
\text { Value }\end{array}$ & $B(95 \% C l)$ & $\begin{array}{l}\text { Standardised } \\
\text { Beta }(95 \% \mathrm{Cl})\end{array}$ & $\begin{array}{l}P \\
\text { value }\end{array}$ \\
\hline $\begin{array}{l}\text { Depression score } \\
\text { mean }(S D),(n=3,242)\end{array}$ & $16.8(5.9)$ & $10.3(7.1)$ & $-0.09(-0.21,0.03)$ & $-0.02(-0.06,0.01)$ & 0.158 & $-0.06(-0.19,0.07)$ & $-0.02(-0.05,0.02)$ & 0.360 \\
\hline $\begin{array}{l}\text { Anxiety score } \\
\text { mean (SD), }(n=3,313)\end{array}$ & $14.3(4.9)$ & $9.1(6.1)$ & $-0.10(-0.21,0.01)$ & $-0.03(-0.06,0.00)$ & 0.078 & $-0.10(-0.21,0.02)$ & $-0.03(-0.06,0.01)$ & 0.094 \\
\hline $\begin{array}{l}\text { Functioning score } \\
\text { mean (SD), }(n=1,782)\end{array}$ & $20.0(8.4)$ & $14.3(9.8)$ & $-0.44(-0.69,-0.20)$ & $-0.08(-0.13,-0.04)$ & $<0.001$ & $-0.46(-0.71,-0.21)$ & $-0.09(-0.14,-0.04)$ & $<0.001$ \\
\hline
\end{tabular}

Adjusted for age, gender, number of sessions and deprivation decile. $n=2$ with missing data on confounders

† Variables recoded so that positive values indicate more improvement in symptoms and negative values indicate less improvement in symptoms

Unstandardised coefficients represents the change in outcome per 1-unit increase in SAPAS score; standardised coefficients represent the SD change in

outcome per SD change in SAPAS score 
Table 5: Association between individual SAPAS items, recovery and reliable improvement

\begin{tabular}{|l|l|l|l|l|}
\hline Treatment Outcomes & \multicolumn{2}{l|}{ Unadjusted } & \multicolumn{2}{l|}{ Adjusted } \\
\cline { 2 - 5 } & OR (95\%CI) & P Value & OR (95\%CI) & $P$ Value \\
\hline Recovery (depression and anxiety) $\dagger$ & & & & \\
Item 1: Difficulty making and keeping friends & $0.51(0.44,0.59)$ & $<0.001$ & $0.64(0.55,0.74)$ & $<0.001$ \\
Item 2: Usually a loner & $0.53(0.47,0.63)$ & $<0.001$ & $0.66(0.56,0.76)$ & $<0.001$ \\
Item 3: Trusting others & $0.60(0.52,0.70)$ & $<0.001$ & $0.78(0.66,0.91)$ & 0.002 \\
Item 4: Normally loses temper easily & $0.74(0.64,0.85)$ & $<0.001$ & $0.89(0.76,1.04)$ & 0.138 \\
Item 5: Normally impulsive & $0.92(0.80,1.06)$ & 0.262 & $1.02(0.88,1.19)$ & 0.755 \\
Item 6: Normally a worrier & $0.70(0.57,0.86)$ & $<0.001$ & $0.80(0.65,0.99)$ & 0.038 \\
Item 7: Depends on others a lot & $0.61(0.52,0.70)$ & $<0.001$ & $0.68(0.58,0.79)$ & $<0.001$ \\
Item 8: Generally a perfectionist & $0.87(0.75,1.00)$ & 0.051 & $0.92(0.79,1.08)$ & 0.311 \\
\hline Reliable improvement (depression or anxiety) & & & & \\
Item 1: Difficulty making and keeping friends & $0.70(0.61,0.82)$ & $<0.001$ & $0.59(0.50,0.69)$ & $<0.001$ \\
Item 2: Usually a loner & $0.75(0.64,0.86)$ & $<0.001$ & $0.65(0.55,0.76)$ & $<0.001$ \\
Item 3: Trusting others & $0.89(0.76,1.03)$ & 0.119 & $0.72(0.62,0.85)$ & $<0.001$ \\
Item 4: Normally loses temper easily & $0.95(0.82,1.10)$ & 0.457 & $0.84(0.72,0.98)$ & 0.030 \\
Item 5: Normally impulsive & $1.10(0.95,1.28)$ & 0.204 & $1.04(0.89,1.21)$ & 0.598 \\
Item 6: Normally a worrier & $0.99(0.81,1.22)$ & 0.940 & $0.83(0.67,1.03)$ & 0.088 \\
Item 7: Depends on others a lot & $0.83(0.72,0.97)$ & 0.018 & $0.76(0.65,0.89)$ & 0.001 \\
Item 8: Generally a perfectionist & $0.92(0.79,1.06)$ & 0.251 & $0.87(0.74,1.02)$ & 0.077 \\
\hline
\end{tabular}

$\dagger$ Recovery among those meeting criteria for clinical caseness for either depression or anxiety at first assessment

Adjusted for age, gender, number of sessions, deprivation decile and baseline depression and anxiety symptom scores. $n=2$ with missing data on confounders OR represents the odds of outcome per 1-unit increase in SAPAS score 
